\title{
Propensity to reappraise promotes resilience to stress-induced negativity bias
}

\author{
Candace M. Raio ${ }^{1}$, Catherine C. Brown ${ }^{2}$ and Maital Neta ${ }^{2^{*}}$
}

${ }^{1}$ New York University Grossman School of Medicine, Neuroscience Institute, New York, NY, USA

${ }^{2}$ University of Nebraska-Lincoln, Department of Psychology, Lincoln, NE, USA

${ }^{*}$ Correspondence concerning this article should be addressed to:

Dr. Maital Neta

Department of Psychology

University of Nebraska-Lincoln

Lincoln, NE, USA

maitalneta@unl.edu

ORCID: https://orcid.org/0000-0001-8586-0963 


\section{Declarations}

\section{Funding}

This work was supported by the National Institutes of Health (NIMH111640; PI: Neta), and by

Nebraska Tobacco Settlement Biomedical Research Enhancement Funds.

\section{Conflicts of Interest}

The authors declare that they have no conflict of interest.

\section{Ethics Approval}

All procedures were approved by the University of Nebraska-Lincoln Committee for the Protection of Human Subjects (IRB approval \#: 20151215793EP) and were in accordance with the 1964 Declaration of Helsinki.

\section{Informed Consent}

All participants gave informed consent before participating in the study.

\section{Data Availability}

Data are available from the corresponding author upon request.

\section{Acknowledgements}

We thank Nicholas R. Harp for assistance with collecting and compiling the data for Study 2. 


\begin{abstract}
Stress exposure is associated with an increased tendency to appraise ambiguous social stimuli as negative. However, it remains unknown whether tendencies to use emotion regulation strategies—such as cognitive reappraisal, which involves altering the meaning of affective stimuli-can buffer these stress effects on social evaluations. Here, we examined whether increased reappraisal use confers resilience against stress-induced negativity bias. In Study 1, healthy participants $(n=43)$ rated the valence of emotionally ambiguous (surprised) faces before and after an acute stress or control manipulation and reported reappraisal habits. Increased negativity ratings were milder for stressed individuals that reported more habitual reappraisal use. In Study 2 ( $n=97)$, we extended this investigation to real-world perceived stress before and during the COVID-19 pandemic. We found that reappraisal tendency moderates the relationship between perceived stress and increased negativity bias. Collectively, these findings suggest that the propensity to reappraise attenuates stress-induced negativity bias when evaluating others under uncertainty.
\end{abstract}

Keywords: stress; reappraisal; ambiguity; negativity bias; COVID-19 


\section{Introduction}

Psychological resilience - defined as an ability to "bounce back from negative emotional experiences" and flexibly adapt to "changing demands of stressful experiences" (Tugade \& Fredrickson, 2004, p. 320) - promotes better life outcomes and overall well-being. For example, high trait resilience is associated with positive indicators of mental health (e.g., life satisfaction, low prevalence of mood disorders; Hu, Zhang, \& Wang, 2015). Moreover, resilient individuals are able to use positive emotions to recover from stressful experiences and adversity (Tugade \& Fredrickson, 2004).

One driving force behind stress resilience is the ability to regulate emotions in a contextually appropriate manner (Waugh, Thompson, \& Gotlib, 2011). Previous studies have supported this association between emotion regulation and resilience to stress: individuals who reported more habitual use of reappraisal, but not suppression (based on scores from the Emotion Regulation Questionnaire; ERQ; Gross \& John, 2003) tended to report less negative mood in response to daily stressors (Johnson et al., 2016). In other words, stressors typically promote negative appraisals of one's environment, but the ability to reappraise may buffer this effect and enhance psychological resilience.

Prior work has found that exposure to stress is associated with more negative perceptions of emotional ambiguity (e.g., surprised faces; Brown, Raio, \& Neta, 2017). Ambiguity is useful for measuring individual differences in emotional bias because it can be readily (and accurately) associated with both positive (e.g., unexpected gift) and negative (e.g., car accident) outcomes. Thus, one's tendency to perceive a negative meaning constitutes one's "valence bias" (Neta, Norris, \& Whalen, 2009). Although there is wide variability in valence bias across individuals, behavioral and neuroimaging studies have supported an initial, bottom-up negative appraisal of ambiguous stimuli (Neta \& Tong, 2016; Petro, Tong, Henley, \& Neta, 2018). It is thought that because stress prioritizes bottom-up processing (Rauch et al., 2000; Hermans et al., 2014), it thereby contributes to a negative valence bias (Brown et al., 2017). 
However, the stress-negativity correspondence is not one-to-one; although some participants undergoing stress experienced increased negativity, others did not. This suggests that, even in the face of the same stressor, these latter individuals may be more psychologically resilient to the effects of stress.

The present studies examined habitual reappraisal and suppression (as measured by $E R Q)$ as a putative buffer against stress-induced negativity. We operationalized resilience as a smaller shift toward negative perceptions of ambiguity after stress, and we predicted that individuals who are more resilient report more habitual reappraisal, but not suppression, under stress. We first explored this question in the context of an existing data set from a study using a laboratory stress manipulation (Study 1) and further examined it in the context of real-world perceived stress imposed by the onset of the COVID-19 pandemic in a second, independent study (Study 2).

\section{Study 1}

\section{Method}

\section{Participants}

The initial sample included 52 participants recruited from the Univeristy of NebraskaLincoln and the surrounding community. An a priori power analysis conducted for our originally published study (Brown et al., 2017) used a between-group comparison of cortisol concentrations after a stressor described in previous work (Raio, Orederu, Palazzolo, Shurick, \& Phelps, 2013). This power analysis idenitifed a minimum of 21 participants per group were necessary to replicate this cortisol comparison with $80 \%$ power, $\alpha=.05$. Given that this was an existing data set, all analyses were constrained to the original sample size reported in our original 2017 paper (Brown et al., 2017). Consistent with these previously published methods, six participants were excluded from the sample for the following reasons: non-normative ratings of clearly-valenced faces $(n=1)$ using the same threshold of minimum $60 \%$ accuracy as in prior 
work (Neta \& Tong, 2016), demonstrating cortisol changes that were more than two standard deviations from the group mean $(n=3)$, providing insufficient saliva for cortisol analysis $(n=1)$, and failing to complete both sessions $(n=1)$. Due to computer error, ERQ data was not recorded for three additional participants. Forty-three participants were included in this report: 20 participants (10 female; age range $=18-27 ; M(S D)$ age $=20.35(2.25)$; race $=20$ White) that were randomly assigned to the stress group, and 23 (11 female, age range $=18-35, M(S D)$ age $=20.04(3.57)$, race $=23$ White) that were randomly assigned to the control group. Participants provided written informed consent at the start of each session. All procedures were approved by the University of Nebraska-Lincoln Committee for the Protection of Human Subjects (IRB approval \#: 20151215793EP) and were in accordance with the 1964 Declaration of Helsinki.

\section{Stimuli}

As in prior work (Neta et al., 2009), stimuli included 48 pictures of faces with either an ambiguous valence (surprise, 24 pictures) or a clear (unambiguous) valence (angry and happy, 12 of each). All expressions were validated by a separate set of participants who labeled each expression; only faces correctly labeled more than $60 \%$ of the time were included. Notably, although some of the surprised expressions might be inherently more positive than others, the critical feature of this task is that all subjects rate the same set of faces, and that there has been shown to be wide variability in ratings across subjects. Fourteen distinct identities were selected from the NimStim standardized facial expression stimulus set (Tottenham et al., 2009), and 20 identities were selected from the averaged Karolinska Directed Emotional Faces database (Lundqvist, Flykt, \& Öhman, 1998). Genders were represented equally, though each identity was not represented in all three expressions.

\section{Procedure}

Participants completed two sessions a week apart, as part of a study to examine the effects of acute stress exposure on valence bias (Brown et al., 2017). On Day 1, participants completed the Emotion Regulation Questionnaire (ERQ), which is a 10-item questionnaire that 
measures the tendency to regulate one's emotions using Cognitive Reappraisal or Expressive Suppression strategies (Gross \& John, 2003). The ERQ measures responses on a 7-point scale ranging from Strongly Disagree to Strongly Agree, where higher numbers indicate increased use of a particular strategy. The ERQ has been shown to have acceptable to excellent levels of internal consistency reliability for both Cognitive Reappraisal (Cronbach's alpha $=0.82-.90)$ and Suppression (alpha $=0.76-.80)$ (Wiltink et al., 2011; Preece et al., 2020) and showed similar reliability estimates in our sample as well (ERQ Reappraisal: alpha $=0.88$; ERQ Suppression: alpha=0.75).

Participants then provided a saliva sample to assess Day 1 baseline cortisol levels and completed the baseline valence bias task. On Day 2, participants provided a saliva sample to assess Day 2 baseline cortisol, followed by a stress manipulation consistent with procedures used by Raio et al. (2013). Specifically, participants in the stress group completed the coldpressor task (Velasco, Gómez, Blanco, \& Rodriguez, 1997), which involved submerging the forearm in ice water $\left(0-4^{\circ} \mathrm{C}\right.$, stress group) for three consecutive minutes. Participants provided a saliva sample 10 minutes after removing their arm from the water (to measure cortisol responses) and then immediately completed the valence bias task. Participants provided another saliva sample 50 minutes after removing their arm from the water, when cortisol levels were expected to return to baseline (to measure the likely recovery response; see Brown et al., 2017), and then completed the valence bias task one final time. Participants in the control group completed the task using warm water $\left(\sim 37^{\circ} \mathrm{C}\right)$; all other saliva sampling procedures were identical to that of the stress group.

Valence bias task. The face stimuli were divided into three subsets of 16 faces. The 16 faces within each subset were presented four times in randomized order, for a total of 64 trials. Participants saw a different subset each of the 3 times they completed the valence bias task, and the order in which each subset was presented (at baseline, 10 minutes post-stressor, or 50 minutes post-stressor) was counterbalanced across all participants. MouseTracker software 
(Freeman \& Ambady, 2010) was used to present the stimuli for $500 \mathrm{~ms}$ each and to record the mouse trajectories of each response as participants rated each face as positive or negative. Participants were instructed to rate the faces as quickly and accurately as possible, and the task did not advance until they made a response. Each trial was followed by as ISI varying from 500 to 8000 ms. Trajectory data were recorded to test hypotheses specifically related to the effects of stress on valence bias and are reported in Brown et al. (2017). Exploratory analyses showed no relationship between mouse trajectories and ERQ scores.

\section{Results}

\section{Stress manipulation check (Cortisol analysis)}

Analysis of cortisol concentrations evidenced an effective stress induction as reported in more detail in our original report (Brown et al., 2017). Briefly, cortisol levels did not differ between the stress and control group on Day 1, when baseline valence bias was measured $(t(41)=-1.15, p=.26)$. On Day 2, a Group (stress, control) $\times$ Time (baseline, 10 min poststressor, 50 min post-stressor) repeated-measures ANOVA revealed a significant Group $\times$ Time interaction $\left(F(2,82)=4.61, p=.013\right.$, partial $\left.\eta^{2}=0.10\right)$. Bonferroni-corrected post-hoc comparisons showed that cortisol at 10 minutes post-stressor was significantly higher for the stress group $(M(S D)=0.32(0.19)$ than the control group $(M(S D)=0.20(0.13), p=.02,95 \%$ confidence interval; $\mathrm{Cl}[0.02,0.22])$, but not at Day 2 baseline $(p=.40,95 \% \mathrm{Cl}[-0.05,0.13])$ or 50 min post-stressor $(p=.20,95 \% \mathrm{Cl}[-.03, .12]$; see Table 1$)$.

\section{Valence Bias}

The dependent measure used for the valence ratings of faces was percent negative ratings, or the percent of trials that a face was rated as negative out of the total number of ratings made for that expression condition (i.e., excluding those on which participants made no response). Valence bias scores for each participant were calculated as the percent negative ratings for the surprised faces only. Consistent with a large body of work examining valence 
bias in response to emotional ambiguity (Neta et al., 2009; Neta et al., 2013; Petro et al., 2018) ratings of happy and angry faces were not analyzed and served solely as a control to ensure that individuals were able to correctly identify clearly-valenced facial expressions. Any deficits in this capacity indicated that surprise ratings may not reflect valence bias, but a broader deficit in facial expression recognition. As expected, participants consistently rated angry faces as negative and happy faces as positive, whereas ratings of surprise varied across individuals (see Table 1), consistent with previous work (Neta et al., 2009). Because we were primarily interested in the stress-related change in ratings, we focused our analyses on valence bias change scores (10 minutes post-stressor minus baseline), as previous work demonstrated a stress-related increase in negative ratings during this time (Brown et al., 2017).

Table 1: Physiological and Behavioral Descriptives

\begin{tabular}{|c|c|c|c|c|c|c|c|c|c|}
\hline & & \multicolumn{2}{|c|}{$\begin{array}{c}\text { Baseline } \\
\text { (Day 1) }\end{array}$} & \multicolumn{2}{|c|}{$\begin{array}{l}\text { Baseline } \\
\text { (Day 2) }\end{array}$} & \multicolumn{2}{|c|}{$\begin{array}{l}10 \text { Minutes Post- } \\
\text { Manipulation }\end{array}$} & \multicolumn{2}{|c|}{$\begin{array}{c}50 \text { Minutes Post- } \\
\text { Manipulation }\end{array}$} \\
\hline & & $M$ & SD & $M$ & SD & $M$ & SD & $M$ & SD \\
\hline \multirow{2}{*}{$\begin{array}{c}\text { Cortisol } \\
\text { Concentrations } \\
\text { ( } \mu \mathrm{g} / \mathrm{dL} \text { ) by } \\
\text { Group }\end{array}$} & Stress & 0.26 & 0.16 & 0.26 & 0.15 & 0.32 & 0.19 & 0.25 & 0.11 \\
\hline & Control & 0.22 & 0.10 & 0.22 & 0.14 & 0.20 & 0.13 & 0.21 & 0.12 \\
\hline \multirow{3}{*}{$\begin{array}{l}\text { Face Ratings } \\
\text { (\% Negativity) } \\
\text { by Expression } \\
\text { Condition }\end{array}$} & Angry & 99.27 & 3.11 & \multicolumn{2}{|c|}{ NA } & 98.98 & 4.90 & 98.98 & 3.59 \\
\hline & Happy & 0.58 & 1.84 & \multicolumn{2}{|c|}{ NA } & 0.73 & 2.44 & 3.34 & 12.38 \\
\hline & Surprise & 68.79 & 20.22 & \multicolumn{2}{|c|}{ NA } & 69.26 & 24.63 & 68.04 & 24.24 \\
\hline
\end{tabular}

\section{Emotion Regulation Strategies and Increased Negativity}

We first conducted two separate regression models predicting the change in valence bias using ERQ Reappraisal and ERQ Suppression as predictors in each of the models. Specifically, we conducted a regression on valence bias change scores with Group (stress, control), ERQ Reappraisal, and their interaction as predictors. There was a significant Group $\times$ ERQ Reappraisal interaction $(\beta=10.39, t(39)=-2.26, p=.03$; unstandardized betas), such that 
greater ERQ Reappraisal scores were negatively associated with valence bias change scores only for the stress group $(\beta=-9.03, t(39)=-2.58, p=.014)$, and not for the control group $(\beta=$ 1.36, $t(39)=.455, p=.65$; Figure 1A). That is, participants in the stress group who reported using reappraisal more often in daily life showed less of a stress-related increase in negative perceptions of ambiguity.

In contrast to the ERQ Reappraisal model, there was no interaction effect in the model that included Group (stress, control), ERQ Suppression, and their interaction as predictors $(\beta=$ $2.47, t(39)=-0.43, p=.67)$. No relationships were observed between valence bias change and the ERQ Suppression score for the control group $(\beta=2.03, t(39)=.42, p=.68)$ or stress group $(\beta=-0.45, t(39)=-.15, p=.88 ;$ Figure 1B). Overall, the ERQ Reappraisal model explained $15.43 \%$ of the variance, and the ERQ Suppression model explained $0.51 \%$ of the variance.

Finally, in order to examine the unique contribution of ERQ Reappraisal on valence bias change (above and beyond that of ERQ Suppression) we conducted a third regression on valence bias change scores including all predictors in the same model: Group (stress, control), ERQ Reappraisal, ERQ Suppression and their two interactions. This analysis revealed a significant effect of ERQ Reappraisal $(\beta=-9.58, t(39)=-2.67, p=.01)$ and a significant Group $\times$ ERQ Reappraisal interaction $(\beta=11.57, t(39)=2.33, p=.025)$, but no effect of ERQ Suppression $(\beta=4.13, t(39)=0.90, p=.38)$ nor a ERQ Suppression $\times$ Group interaction $(\beta=-$ $5.43, t(39)=-0.97, p=.34)$. These results support our prediction of a selective relationship between reappraisal - but not suppression - and valence bias change, and suggest that this effect was moderated by group such that the effect was only significant for those undergoing a stress induction. 


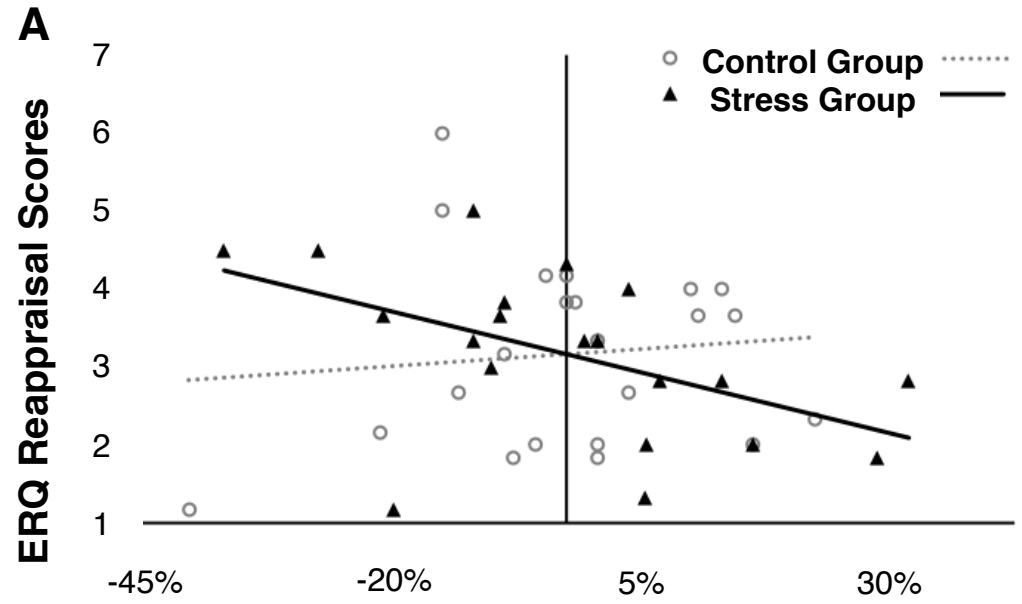

(more positive post-stressor)

Change in Valence Ratings of Surprise Pre- to Post-Stressor

B

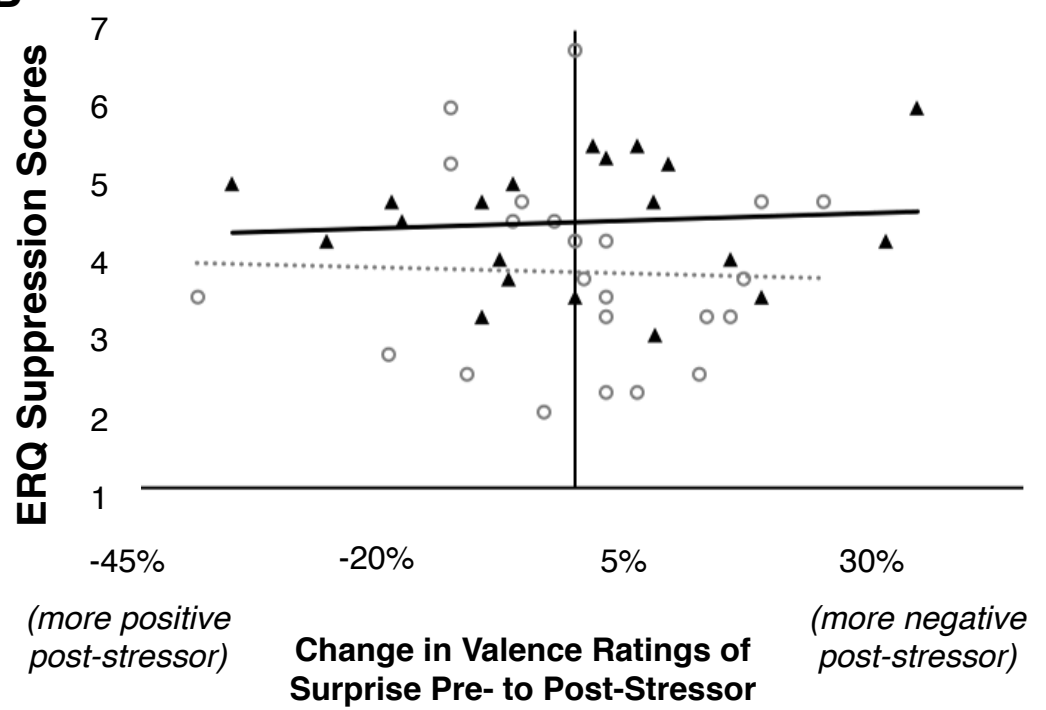

Figure 1: Relationship between reappraisal and stress-related changes in valence ratings of surprised faces. (A) The stress-related change in valence ratings was negatively related with the ERQ Reappraisal score in the stress group $(\beta=-9.03, t(39)=$ $-2.58, p=.014)$ but not (more negative post-stressor)

controls $(\beta=1.36, t(39)$

$=.455, p=.65)$, such that participants in the stress group who used reappraisal more habitually showed less of a stress-related increase in negativity. (B) The stressrelated change in valence ratings showed no significant relationship with the $E R Q$ Suppression score in either group ( $p s>.67)$.

\section{Emotion Regulation Strategies and Increased Cortisol}

We conducted similar regression models predicting change in cortisol levels (rather than change in valence bias) from Day 2 baseline to post-stressor, using ERQ Reappraisal and ERQ Suppresion, respectively. No interaction effects emerged in the model using Group (stress, control), ERQ Reappraisal, and their interaction as predictors $(\beta=-0.01, t(39)=-1.57, p=.13)$, nor in the model with Group (stress, control), ERQ Suppression, and their interaction as predictors $(\beta=-0.01, t(39)=-0.80, p=.43)$. 


\section{Study 2}

Study 1 revealed that greater use of reappraisal to regulate one's emotions confers a buffering effect on increased negativity bias after exposure to stress. The fact that stress exposure promotes negative appraisals of ambiguous stimuli is consistent with a emerging body of empirical work (Starcke \& Brand, 2016). However, our initial assessment of this relationship occurred within a controlled laboratory setting and in a relatively small sample. Thus, to probe whether such an effect persists in real-world stressful contexts, and to directly test if reappraisal use moderates the effects of stress on negativity bias, we next sought to replicate and extend our findings in an independent sample experiencing stress during the onset of the COVID-19 pandemic.

\section{Method}

\section{Participants}

Participants were recruited for an online study that measured valence bias before and after the start of the COVID-19 pandemic, using Amazon's Mechanical Turk (M-Turk; Horton, Rand \& Zeckhause, 2011). Of the 229 participants included in the initial wave of data collection before the onset of the pandemic (Harp et al., 2020; 122 female; age range = 18-76 years; mean(SD) age $=44.77(14.43)$; race distribution: 15 Asian, 20 Black, 177 White, 5 Other, and 12 Unknown), 110 participants volunteered to participate in a follow-up after the onset of the pandemic. According to an a priori power analysis, a minimum of 77 participants were necessary for a moderation analysis with $80 \%$ power, $\alpha=.05$ and effect size of 0.15 .

As in Study 1 and previous work (Neta \& Whalen, 2010; Neta et al., 2013; Neta \& Tong, 2016), eleven participants were excluded from the follow-up sample for non-normative ratings of clearly-valenced faces (i.e., accuracy for valence ratings of angry and/or happy faces was below $60 \%$ ) to ensure an accurate representation of the bias in response to ambiguity. Further, two participants were removed for scoring more than 3 SDs below the mean on the ERQ

(Reappraisal), since this was the primary construct of interest and would serve as our moderator 
variable. Our final sample consisted of 97 participants (53 female; age range at the first session = 21-76; $M(S D)$ age = 47.58(13.73); race distribution: 5 Asian, 8 Black, 76 White, 1 Other, and 7 Unknown). Participants provided informed consent at the start of each session. All procedures were approved by the University of Nebraska-Lincoln Committee for the Protection of Human Subjects (IRB approval \#: 20200520425EP) and were in accordance with the 1964 Declaration of Helsinki.

\section{Stimuli}

Three task blocks (faces, scenes, and words) were used to assess valence bias. As in previous work (Neta et al., 2013), the face and scene task blocks included 24 ambiguous images and 24 clear images (12 positive and 12 negative). The facial expressions were selected from the NimStim (Tottenham et al., 2011) and Karolinska Directed Emotional Faces (Lundqvist et al., 1998) sets, and the scenes were selected from the International Affective Picture System (IAPS; Lang et al., 2008). For the words block, the 32 ambiguous, 16 positive, and 16 negative words were used (Harp et al., 2020). However, for the purposes of this experiment in replicating and extending findings from Study 1 that used only face stimuli, here we focus only on responses to the face blocks. As in Study 1, ratings of happy and angry faces were not of interest above and beyond serving as anchors to ensure a reliable measure of valence bias under emotional ambiguity (i.e., surprise).

\section{Procedure}

Participants completed two sessions, one between October 2019 and January 2020 (before the onset of the COVID-19 pandemic) and a second session between April and May 2020 (after the pandemic onset), as part of a study to understand the effects of real-world stress on valence bias. During Session 1, participants completed the valence bias task on face, scene, and word stimuli, where each stimulus category was presented in separate blocks (2 total blocks for each category). The order of blocks was counterbalanced across participants, but one block of each category was always presented before presenting the second block for each 
category (Harp et al., 2020). Each block included 50\% stimuli that were ambiguous, and 50\% that were clearly valenced ( $25 \%$ clearly positive and $25 \%$ clearly negative), as in previous work (Neta et al., 2013). Note that, for the purposes of this report in extending the findings from Brown et al. (2017), we focus on the valence rating responses to ambiguous face stimuli (clear stimuli were presented primarily to ensure that participants were performing the task accurately). During Session 2, participants completed the same valence bias task again in a new counterbalanced order, and then completed a series of self-report surveys including the Emotion Regulation Questionnaire (ERQ) (Gross \& John, 2003). As in Study 1, the ERQ showed adequate reliability in our sample for both ERQ Reappraisal (alpha $=0.86$ ) and ERQ Suppression (alpha=0.83).

Participants also completed the Perceived Stress Scale (PSS; Cohen, Kamarck, \& Mermelstein, 1983), a widely-used and well-validated measure of self-reported perceived stress experienced over the previous month. The PSS is a 10-item questionnaire where perception of stress is measured by probing how uncontrollable, unpredictable and overloaded participants have felt over the last month, with responses provided on a 4-point scale ranging from Never to Very Often. The PSS has been found to have acceptable to excellent levels of internal consistency reliability ( $\alpha=.78$; Cohen $\&$ Williamson, 1988; $\alpha=.87$; Baik et al., 2019) and showed good reliability in our sample (alpha $=0.92)$. Self-report surveys were administered after the face ratings since valence bias is known to be highly sensitive to transient changes in affective state (Brown et al., 2017; Neta et al., 2017; Neta, Davis, \& Whalen, 2011) and we wanted to avoid any priming effects that could potentially arise from the affective scales participants were completing.

\section{Results}

Given that reappraisal tendency buffered stress-induced increases in negativity bias in Study 1, here we examined the extent to which this effect generalized to real-world stressful contexts (i.e., perceived stress during the COVID pandemic). We capitalized on the continuous nature of 
participants' perceived stress scores-unike Study 1, where stress group assignment was binary—by testing whether reappraisal tendency moderated the effect of perceived stress on change in negativity bias. Our moderation model was conducted using the PROCESS SPSS macro (Hayes, 2013), where change in negativity bias (a difference score in ratings of surprised faces after $>$ before the onset of the COVID pandemic) was included as the outcome variable, with Perceived Stress Scale (PSS) scores as the predictor and ERQ Reappraisal as a moderator of the relationship between these variables. Both ERQ Reappraisal and Perceived Stress were mean-centered prior to analysis. Table 2 reports the descriptive statistics for each variable.

Table 2: Descriptive statistics of observed variables

\begin{tabular}{|c|c|c|c|}
\hline & $M(S D)$ & $\begin{array}{c}\text { Sample } \\
\text { Range }\end{array}$ & $\begin{array}{c}\text { Possible } \\
\text { Range }\end{array}$ \\
\hline $\begin{array}{c}\text { Change in negativity bias (valence } \\
\text { bias ratings after > before the onset of } \\
\text { the COVID pandemic) }\end{array}$ & $\begin{array}{c}11.75 \% \\
(29.62)\end{array}$ & $\begin{array}{c}-52.17- \\
+100 \%\end{array}$ & $-100-+100 \%$ \\
\hline Reappraisal score (ERQ-R) & $5.18(1.03)$ & $1.83-7$ & $1-7$ \\
\hline Perceived Stress score (PSS) & $15.16(7.96)$ & $1-32$ & $0-40$ \\
\hline
\end{tabular}

${ }^{*}$ Note: Negative values in change in negativity bias denote a shift toward more positive ratings of surprised faces after compared to before the onsets of the COVID-19 pandemic; positive values denote a shift toward more negative ratings.

The overall model was significant in accounting for $7.8 \%$ of the variance $(F(3,93)=2.61$, $p=.05)$. The interaction uniquely accounted for $5.2 \%$ of the variance $(F(1,93)=5.26, p=.02)$, indicating that the relationship between change in negativity bias and perceived stress was indeed moderated by reappraisal tendency (ERQ Reappraisal). The conditional effect of Perceived Stress on ERQ Reappraisal was significant at one standard deviation below the mean of cognitive reappraisal $(b=.12,95 \% \mathrm{Cl}[0.0328,0.2043], t(93)=2.75, p=.007)$, but not at the mean $(b=.05,95 \% \mathrm{Cl}[-0.0152,0.1061], t(93)=1.49, p=.14)$, or at one standard deviation above the mean $(b=-.03,95 \% \mathrm{Cl}[-0.1171,0.0619], t(93)=-.61, p=.54)$. 
To probe the significant interaction, we conducted a regions of significance analysis such that conditional effects of perceived stress on change in negativity bias were estimated at all observed levels of ERQ Reappraisal (i.e., scores ranging from 1.83 to 7), and the significance of those conditional effects were examined. Perceived stress was associated with a greater increase in negativity bias for individuals with an ERQ Reappraisal scores -.22 below the mean and lower (i.e., scores ranging from 1.83 to 4.96; see Figure 2). That is, participants experiencing higher perceived stress after the onset of the COVID pandemic who also report lower reappraisal tendency showed more of a stress-related increase in negative perceptions of ambiguity.

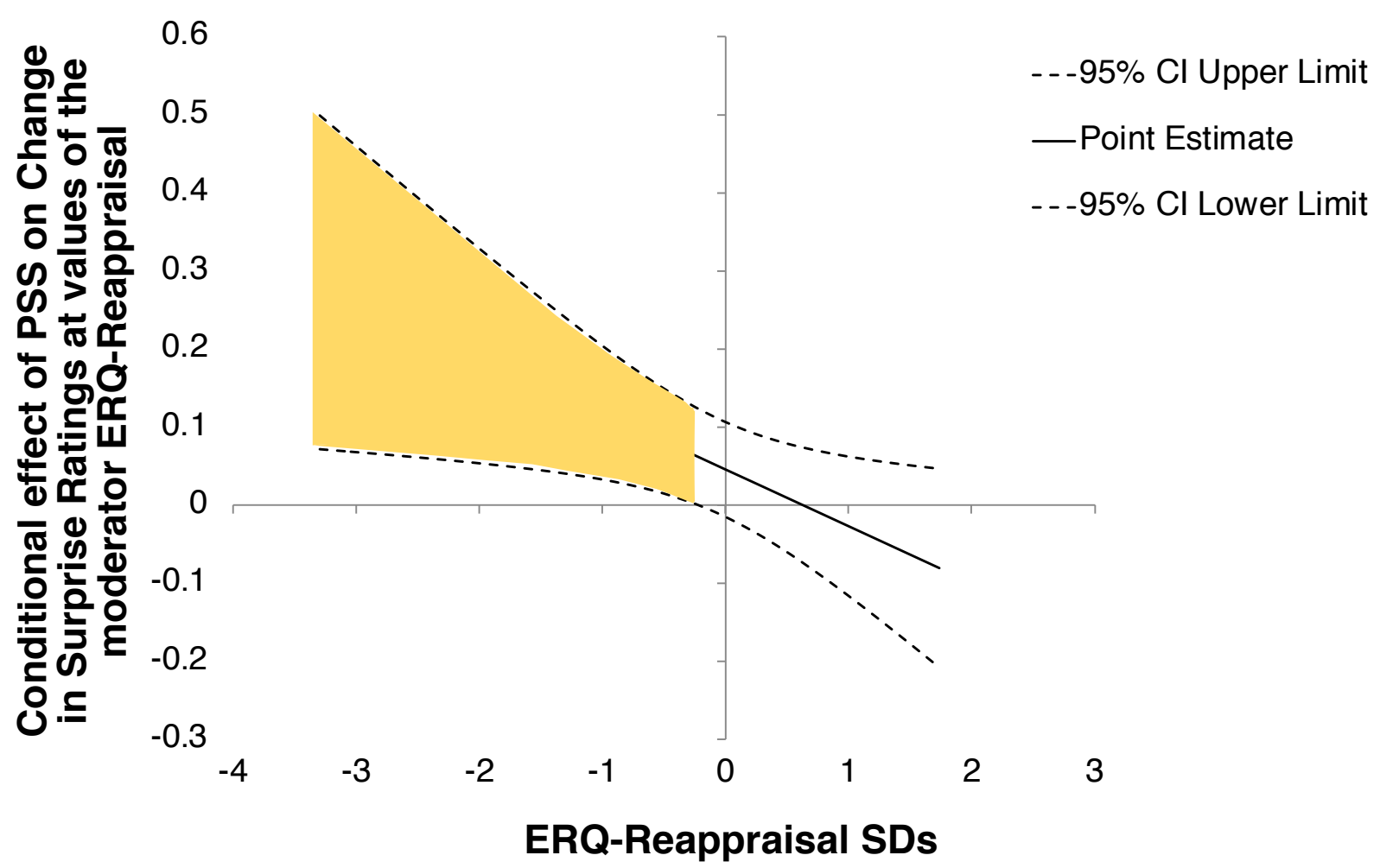

Figure 2: Relationship between valence bias and perceived stress as a function of ERQ-R score. The conditional effect of perceived stress during the COVID pandemic on valence bias change is plotted for a range of ERQ-R scores. In individuals with low reappraisal tendency, stress increased negative ratings of ambiguous facial expressions, while in individuals with high reappraisal tendency, this stress-related increase in negativity bias was not observed. 
We next assessed whether a similar effect was observed using suppression tendency (ERQ-S) rather than reappraisal. A comparable moderation analysis was not significant $(F(3$, $93)=0.913, p=0.44)$, and revealed no significant interaction between ERQ suppression and Perceived Stress $(b=0.005,95 \% \mathrm{Cl}[-0.0604,0.0615], t=0.02, p=.98)$. In other words, ERQ suppression does not appear to moderate the relationship between perceived stress and valence bias change.

\section{Discussion}

Results from this work demonstrate an important role for reappraisal tendencies as a buffer from stress-related negativity. Across two independent studies, we found that individuals who habitually reappraise appear less vulnerable to stress-related increases in negative perceptions of ambiguituous social stimuli, compared to those who report reappraising less habitually or those using alternative regulatory strategies (suppression). We also replicated these findings outside a laboratory setting in the context of a real-world stressor-the COVID-19 pandemic. These findings suggest that those who more often engage in reappraisal may experience a milder impact of stress (Jamieson, Nock, \& Mendes, 2012) on valence bias, which may confer resilience by prompting a less negative emotional experience following stress (Fredrickson, Tugade, Waugh, \& Larkin, 2003). These findings are also consistent with recent work showing that cognitive forms of emotion regulation mediated the association between ventromedial prefrontal cortex activity during a stressor and more positive emotions during stress recovery (Yang et al., 2018). We propose that this effect points to a mechanism whereby individuals in the practice of regulating potential negativity through reappraisal may more spontaneously override the initial negative appraisals that arise when confronting ambiguity and, more specifically, override negativity biases driven by stress exposure.

In Study 1, although habitual reappraisal mitigated the negative behavioral consequences of stress, it showed no impact on physiological stress reactivity (changes in 
cortisol following a stressor). Interestingly, the literature shows inconsistent findings relating reappraisal and cortisol reactivity. For example, some studies demonstrate that reappraisal during a laboratory-induced stressor is associated with greater cortisol reactivity, while others showed no effect or have found that habitual reappraisal was associated with less cortisol reactivity (see Krkovic, Clamor, \& Lincoln, 2018 for a review). Notably, the latter finding measures responses to a stressor that was qualititatively different from laboratory-based inductions in that it was voluntary (i.e., skydiving). Indeed, participants who volunteer for such an unpredictable and stressful experience may already appraise ambiguity in a more positive light to begin with (Crum, Jamieson, \& Akinola, 2020). These strategies, when activated during an acute stressor, could help them arrive at positive perceptions of ambiguity.

In contrast to Study 1, in which stress exposure was experimentally induced, Study 2 assessed whether this buffering effect of reappraisal tendency generalized to a real-world stessor. By measuring valence bias change in participants before and after the start of the COVID pandemic, we were able to leverage the effects of a widespread societal stressor on participants that varied depending on each individuals' subjectively perceived stress. This afforded the opportunity to test whether these results replicated using a continuous measure of stress exposure occurring in participants' daily lives, rather than within a laboratory setting. In doing so, we observed that habitual reappraisal moderated the effect of perceived stress on the change in bias, such that lower reappraisal revealed an increase in perceptions of negativity under higher perceived stress, while higher reappraisal buffered this effect. In contrast, habitual use of expressive suppression to regulate emotion did not show the same pattern of results. Collectively, these results suggest that habitual use of cognitive strategies to deliberately change one's emotional state (reappraisal) may generalize to attenuating stress-induced negativity bias of social stimuli.

A number of limitations should be noted for future work. Study 1 was constrained to the existing sample size of our originally published study (Brown et al., 2017), thus future work 
should seek to replicate these effects in larger samples, and using different stress manipulation techniques. Study 2 was conducted using a longitudinal design, rendering it difficult to retain our entire original sample size (although we note our final sample was adequately powered to observe our predicted effect). Study 2 was also conducted online, which afforded us the opportunity to reach a more representative sample of individuals affected by the COVID pandemic. However, we note that our final sample was heavily skewed toward white participants, thus further research should investigate whether these results generalize to more diverse samples. Finally, although those participants who endorsed higher reappraisal scores showed a buffering effect of stress on valence bias, we cannot conclude that these participants definitively used this strategy when rating surprised faces. Thus, a final important avenue for future research will be to determine the relationship between deliberate or instructed emotion regulation strategies and individual differences in valence bias, particularly when faced with a variety of stressors.

Open Practices Statement. Neither of the experiments reported in this article was formally preregistered. Neither the data nor the materials have been made available on a permanent third-party archive; requests for the data or materials can be sent via email to the senior author.

Author Contributions. All authors contributed to the study design. Data collection were performed by C.C. Brown. All authors performed data analysis, wrote the manuscript and have approved the final version of the manuscript for submission. 


\section{References}

Brown, C. C., Raio, C. M., \& Neta, M. (2017). Cortisol responses enhance negative valence perception for ambiguous facial expressions. Scientific Reports, 7(1), 15107. https://doi.org/10.1038/s41598-017-14846-3

Crum, A. J., Jamieson, J. P., \& Akinola, M. (2020). Optimizing stress: An integrated intervention for regulating stress responses. Emotion, 20(1), 120-125.

\section{https://doi.org/10.1037/emo0000670}

Fredrickson, B. L., Tugade, M. M., Waugh, C. E., \& Larkin, G. R. (2003). What good are positive emotions in crisis? A prospective study of resilience and emotions following the terrorist attacks on the United States on September 11th, 2001. Journal of Personality and Social Psychology, 84(2), 365-376. https://doi.org/10.1037/0022-3514.84.2.365

Freeman, J. B., \& Ambady, N. (2010). MouseTracker: Software for studying real-time mental processing using a computer mouse-tracking method. Behavior Research Methods, 42(1), 226-241. https://doi.org/10.3758/BRM.42.1.226

Gross, J. J., \& John, O. P. (2003). Individual differences in two emotion regulation processes: Implications for affect, relationships, and well-being. Journal of Personality and Social Psychology, 85(2), 348-362. https://doi.org/10.1037/0022-3514.85.2.348

Harp, N., Brown, C., \& Neta, M. (2020, June 3). Spring break or heart break? Extending valence bias to emotional words. https://doi.org/10.31234/osf.io/mrg3t

Hayes, A. F. (2013). PROCESS SPSS Macro [Computer software andmanual].

Hermans, E. J., Henckens, M. J., Joëls, M. \& Fernández, G. (2014) Dynamic adaptation of large-scale brain networks in response to acute stressors. Trends Neurosci 37, 
304-14.

Horton, J. J., Rand, D. G. \& Zeckhauser, R. J. (2011) The online laboratory: conducting 599 experiments in a real labor market. Exp. Econ. 14, 399-425.

Hu, T., Zhang, D., \& Wang, J. (2015). A meta-analysis of the trait resilience and mental health. Personality and Individual Differences, 76, 18-27. https://doi.org/10.1016/j.paid.2014.11.039

Jamieson, J. P., Nock, M. K., \& Mendes, W. B. (2012). Mind over matter: Reappraising arousal improves cardiovascular and cognitive responses to stress. Journal of Experimental Psychology: General, 141(3), 417-422. https://doi.org/10.1037/a0025719

Johnson, J., O’Connor, D. B., Jones, C., Jackson, C., Hughes, G. J., \& Ferguson, E. (2016). Reappraisal buffers the association between stress and negative mood measured over 14 days: Implications for understanding psychological resilience. European Journal of Personality, 30(6), 608-617. https://doi.org/10.1002/per.2080

Krkovic, K., Clamor, A., \& Lincoln, T. M. (2018). Emotion regulation as a predictor of the endocrine, autonomic, affective, and symptomatic stress response and recovery. Psychoneuroendocrinology, 94, 112-120.

https://doi.org/10.1016/j.psyneuen.2018.04.028

Lundqvist, D., Flykt, A., \& Öhman, A. (1998). The Karolinska Directed Emotional Faces—KDEF (CD ROM). Stockholm: Karolinska Institute, Department of Clinical Neuroscience, Psychology Section.

Neta, M., Davis, F. C., \& Whalen, P. J. (2011). Valence resolution of facial expressions using an emotional oddball task. Emotion, 11(6), 1425-1433.

Neta, M., Cantelon, J., Mahoney, C. R., Taylor, H. A., \& Davis, F. C. (2017). The impact of uncertain threat on affective bias: Individual differences in response to ambiguity. Emotion, 17(8):1137-1143. doi: 10.1037/emo0000349. 
Neta, M., Norris, C. J., \& Whalen, P. J. (2009). Corrugator muscle responses are associated with individual differences in positivity-negativity bias. Emotion, 9(5), 640-648. https://doi.org/10.1037/a0016819

Neta, M., Kelley, W. M., \& Whalen, P. J. (2013). Neural responses to ambiguity involve domaingeneral and specific emotion processing systems. Journal of Cognitive Neuroscience, 25(4), 547-557.

Neta, M., \& Tong, T. T. (2016). Don't like what you see? Give it time: Longer reaction times associated with increased positive affect. Emotion, 16(5), 730-739. https://doi.org/10.1037/emo0000181

Petro, N. M., Tong, T. T., Henley, D. J., \& Neta, M. (2018). Individual differences in valence bias: FMRI evidence of the initial negativity hypothesis. Social Cognitive and Affective Neuroscience, 13(7), 687-698. https://doi.org/10.1093/scan/nsy049

Raio, C. M., Orederu, T. A., Palazzolo, L., Shurick, A. A., \& Phelps, E. A. (2013). Cognitive emotion regulation fails the stress test. Proceedings of the National Academy of Sciences, 110(37), 15139-15144. https://doi.org/10.1073/pnas.1305706110

Rauch, S. L., Whalen, P. J., Shin, L. M., Mclnerney, S. C., Macklin, M. L., Lasko, N. B., Orr, S. P., \& Pitman, R. K. (2000). Exaggerated amygdala response to masked facial stimuli in posttraumatic stress disorder: A functional MRI study. Biological Psychiatry, 47(9), 769_ 776. https://doi.org/10.1016/S0006-3223(00)00828-3

Starcke, K. \& Brand, M. (2016) Effects of stress on decisions under uncertainty: a metaanalysis. Psychol Bull 142, 909-33.

Tottenham, N., Tanaka, J. W., Leon, A. C., McCarry, T., Nurse, M., Hare, T. A., Marcus, D. J., Westerlund, A., Casey, B., \& Nelson, C. (2009). The NimStim set of facial expressions: Judgments from untrained research participants. Psychiatry Research, 168(3), 242-249. https://doi.org/10.1016/j.psychres.2008.05.006 
Tugade, M. M., \& Fredrickson, B. L. (2004). Resilient individuals use positive emotions to bounce back from negative emotional experiences. Journal of Personality and Social Psychology, 86(2), 320-333. https://doi.org/10.1037/0022-3514.86.2.320

Velasco, M., Gómez, J., Blanco, M., \& Rodriguez, I. (1997). The cold pressor test: Pharmacological and therapeutic aspects. American Journal of Therapeutics, 4(1), 3438. https://doi.org/10.1097/00045391-199701000-00008

Waugh, C. E., Thompson, R. J., \& Gotlib, I. H. (2011). Flexible emotional responsiveness in trait resilience. Emotion, 11(5), 1059-1067. https://doi.org/10.1037/a0021786

Yang, X., Garcia, K. M., Jung, Y., Whitlow, C. T., McRae, K., \& Waugh, C. E. (2018). VmPFC activation during a stressor predicts positive emotions during stress recovery. Social Cognitive and Affective Neuroscience, 13(3), 256-268.

https://doi.org/10.1093/scan/nsy012 